Article

\title{
Using Class Probabilities to Map Gradual Transitions in Shrub Vegetation from Simulated EnMAP Data
}

\section{Stefan Suess *, Sebastian van der Linden, Akpona Okujeni, Pedro J. Leitão, Marcel Schwieder and Patrick Hostert}

Geography Department, Humboldt-Universität zu Berlin, Unter den Linden 6, D-10099, Berlin, Germany; E-Mails: sebastian.linden@geo.hu-berlin.de (S.L.); akpona.okujeni@geo.hu-berlin.de (A.O.); p.leitao@geo.hu-berlin.de (P.J.L.); marcel.schwieder@geo.hu-berlin.de (M.S.); patrick.hostert@geo.hu-berlin.de (P.H.)

* Author to whom correspondence should be addressed; E-Mail: stefan.suess@ geo.hu-berlin.de; Tel.: +49-30-2093-6894; Fax: +49-30-2093-6848.

Academic Editors: Saskia Foerster, Lenio Soares Galvao and Prasad S. Thenkabail

Received: 3 May 2015 / Accepted: 12 August 2015 / Published: 18 August 2015

\begin{abstract}
Monitoring natural ecosystems and ecosystem transitions is crucial for a better understanding of land change processes. By providing synoptic views in space and time, remote sensing data have proven to be valuable sources for such purposes. With the forthcoming Environmental Mapping and Analysis Program (EnMAP), frequent and area-wide mapping of natural environments by means of high quality hyperspectral data becomes possible. However, the amplified spectral mixing due to the sensor's ground sampling distance of $30 \mathrm{~m}$ on the one hand and the patterns of natural landscapes in the form of gradual transitions between different land cover types on the other require special attention. Based on simulated EnMAP data, this study focuses on mapping shrub vegetation along a landscape gradient of shrub encroachment in a semi-arid, natural environment in Portugal. We demonstrate how probability outputs from a support vector classification (SVC) model can be used to extend a hard classification by information on shrub cover fractions. This results in a more realistic representation of gradual transitions in shrub vegetation maps. We suggest a new, adapted approach for SVC parameter selection: During the grid search, parameter pairs are evaluated with regard to the prediction of synthetically mixed test data, representing shrub to non-shrub transitions, instead of the hard classification of original, discrete test data. Validation with an unbiased, equalized random sampling shows that the resulting shrub-class probabilities from adapted SVC more accurately
\end{abstract}


represent shrub cover fractions (mean absolute error/root mean squared error of $16.3 \% / 23.2 \%)$ compared to standard SVC (17.1\%/29.5\%). Simultaneously, the discrete classification output was considerably improved by incorporating synthetic mixtures into parameter selection (averaged F1 accuracies increased from $72.4 \%$ to $81.3 \%$ ). Based on our findings, the integration of synthetic mixtures into SVC parameterization allows the use of SVC for sub-pixel cover fraction estimation and, this way, can be recommended for deriving improved qualitative and quantitative descriptions of gradual transitions in shrub vegetation. The approach is therefore of high relevance for mapping natural ecosystems from future EnMAP data.

Keywords: class probabilities; EnMAP; imaging spectroscopy; Portugal; shrub ecosystem; sub-pixel mapping; support vector classification

\section{Introduction}

Natural and semi-natural ecosystems play an important role in conserving biodiversity and providing essential ecosystem services. Shrub-dominated ecosystems, for example, sequester carbon, regulate climate, provide biomass, offer habitats for organisms, and may protect soils, control fire regimes, or improve water quality [1,2]. Natural areas in the Mediterranean or semi-arid regions are often covered by shrub vegetation [3]. The Mediterranean region - due to its intensive land use history - is highly susceptible to land degradation [4]. Formerly cultivated land is abandoned, leading to the subsequent succession of natural vegetation and shrub encroachment, a phenomenon widely reported during the past decades [5-7]. Such spatio-temporal change processes lead to landscape patterns of gradual transitions in shrub vegetation, with different stages of shrub development (gradients from very young and small to mature and high) and varying degrees of densities (gradients from sparse to high dense), accompanied by changing portions of additional ground vegetation and background cover types, such as trees, herbaceous plants, rocks, and bare soil. The mapping and monitoring of these natural ecosystems and ecosystem transitions is hence very challenging. However, such efforts are crucial to develop both a better understanding of land change processes and sustainable management strategies, which ultimately supports the provision of ecosystem services.

By providing synoptic views in space and time, remote sensing data are a major source for extensively mapping and monitoring natural environments at a high frequency [8-10]. Particularly multispectral imagery at $30 \mathrm{~m}$ spatial resolution from the Landsat satellites constitute a typical data source for extensive shrub cover maps $[11,12]$. Other studies make use of multispectral data with high spatial resolution $(<10 \mathrm{~m})$ for the spatial and temporal characterization of shrub vegetation $[13,14]$. However, especially in semi-arid environments such as the Mediterranean region, when mapping shrub cover with varying densities, development stages, and background cover types, the use of multispectral information may be limited. Mapping efforts are impeded by the low spectral contrast between different land cover types when adopting broadband reflectance data. In contrast, hyperspectral data enhance the distinction of different land cover types by providing hundreds of contiguous spectral bands $[15,16]$. The potential of data with high spectral information content for the thematically detailed mapping of shrub species 
was demonstrated in various studies [17-19]. Yet most hyperspectral-based analyses are constrained to airborne data with limited spatial coverage acquired during sporadic flight campaigns, thus restricting the operational mapping and monitoring opportunities. A few studies used spaceborne hyperspectral data (e.g., from the Compact High Resolution Imaging Spectrometer or the Hyperion instrument) for shrub vegetation mapping [16,20-22]. However, the hyperspectral data availability will change with the advent of new operational spaceborne imaging spectrometers, such as the Environmental Mapping and Analysis Program hyperspectral imager (EnMAP, [23]) or the Hyperspectral Infrared Imager (HySPIRI, [24]). The EnMAP sensor will frequently capture high quality hyperspectral imagery with regional coverage and a $30 \mathrm{~m}$ ground sampling distance (g.s.d.), and this way becomes an unique data source promoting innovative environmental applications [25].

Land cover classifications (also referred to as hard classification) are one of the most commonly used analyses of remote sensing data [26,27]. Many procedures for generating accurate land cover maps exist, and discrete maps are both easy to interpret and sufficient for many subsequent applications. Nevertheless, hard per-pixel classifications considerably generalize the true land cover for two reasons. First, they do not account for spectral mixing between different land cover types within single pixels. The extent of spectral mixing therefore depends on the composition and fragmentation of the environment [16,28,29], and increases with decreasing spatial resolution of the sensor. Second, they often fail to realistically represent landscapes which are not characterized by sharp boundaries but by gradual transitions, as it is typical for natural environments [30-32]. As a consequence, quantitative methods, for example, multiple endmember spectral mixture analysis [33] or regression approaches [34], increasingly gained interest as alternatives as they directly provide information on sub-pixel land cover fractions. Despite the good results of these quantitative methods, hard classification remains frequently used, mainly because for many land cover types and in many applications the discrete information is sufficient and the collection of training information is relatively simple. In this context, it seems useful to advance easy-to-use classification approaches in a way that they ideally enable an accurate hard classification of the dominant land cover while generating cover fraction estimates for those land cover types that are characterized by gradual landscape transitions on-the-fly.

Machine learning-based classification became a widely used technique for the processing of remote sensing data [35]. One of the most prominent examples is the kernel-based support vector classification (SVC), which was proven a very accurate and reliable technique, successfully applied in a wide range of domains [36,37], and particularly suited for high-dimensional data [38-40]. Common implementations of SVC use posterior class probabilities as an intermediate step on the way to the discrete class decision[41,42]. The continuous information in these probabilities was shown to be of high value for complex classification tasks [43]. However, only few examples exist where probabilities were related to land cover fractions to better characterize mixed pixels $[44,45]$. This is due to the fact that these probabilities do not necessarily correlate with cover fraction estimates [46], such as estimates from unmixing or regression approaches. Instead, they are derived through model optimization with regard to the hard discrimination of classes. In a recent study, a new strategy for the parameter selection of classification approaches to directly relate class probabilities to cover fraction estimates was introduced [46]. The authors adapt class probabilities by integrating quantitative data into parameter selection, i.e., during the grid search, parameter pairs are evaluated with regard to the prediction of synthetically mixed test data representing cover fractions instead of the hard classification of original, discrete test data. Synthetic 
mixtures are directly calculated from all input training samples by gradual, step-wise linear mixing and no further user input or interaction is required. The efficiency and stability of this strategy was demonstrated using the kernel-based import vector machine classifier and field spectra. However, tests on actual image data with more complex mixtures as, for example, expected from future spaceborne EnMAP data are still lacking. At the same time, it seems adequate to evaluate the advantages of synthetic mixtures in the parameterization process of machine learners in SVC, one of the strongest, most frequently used classifiers in the field of remote sensing.

The overarching goal of this work is to demonstrate how probability outputs from a SVC can be used to extend a hard classification (qualitative) by information on cover fractions (quantitative) when mapping gradual transitions of shrub vegetation with simulated EnMAP data from a natural environment in Portugal. We aim at illustrating that little changes in parameterization can lead to better cover fraction representations by class probabilities while simultaneously improving the hard classification output. To evaluate this strategy, our specific objectives are to compare (1) the accuracies of the hard classification; and (2) the sub-pixel cover fraction estimates of shrub cover derived from the standard SVC implementation and the adapted SVC approach. We additionally provide a comprehensive analysis and discussion on the strengths and weaknesses of our approach, exemplified for four spatial subsets representing the typical landscape patterns of gradual transitions in shrub vegetation. Our study contributes to bridging between qualitative and quantitative mapping, which is of particular relevance when adopting forthcoming spaceborne hyperspectral data for mapping assessment in natural ecosystems.

\section{Study Area and Materials}

\subsection{Study Area}

The study area is located between the towns of Castro Verde and Mértola in southern Portugal (Figure 1a). The slightly undulating region reflects the typical structure of a semi-arid, natural Mediterranean environment, with land cover types such as cereal fields, fallow grasslands, woodlands, and shrublands [47], as illustrated in Figure 2. The extensive agricultural cultivation of cereals in the region's land use history has led to a spatio-temporal landscape mosaic, with dominant fallow grasslands and winter cereal crops, and ploughed and stubble fields [48,49]. During the last decades these steppe-like landscapes have changed due to agricultural intensification, land abandonment, and the afforestation ( $c f$. Figure 2c) [49] of eucalyptus (Eucalyptus globulus), umbrella pine (Pinus pinea), and holm oak (Quercus rotundifolia). Agricultural land abandonment has led to increasing shrub encroachment on fallow lands, which is particularly visible in the southeastern part of the study area. In contrast, the northwestern part benefits from management incentives, which promote extensive agriculture use. This has caused spatially fragmented patterns of rockrose (Cistus spp.) patches ( $c f$. Figure 2), which are common pioneer shrubs in that region. The study area comprises an observed landscape gradient of increasing shrub encroachment along a northwest- to southeast-oriented transect of about 30 by $7 \mathrm{~km}$. In addition to the entire gradient, this work considers four spatial subsets representing the typical landscape patterns of gradual transitions in shrub vegetation, including high and medium dense shrub areas, shrub in tree plantations, and early successional shrub sites (Figure 1b). 
(a)

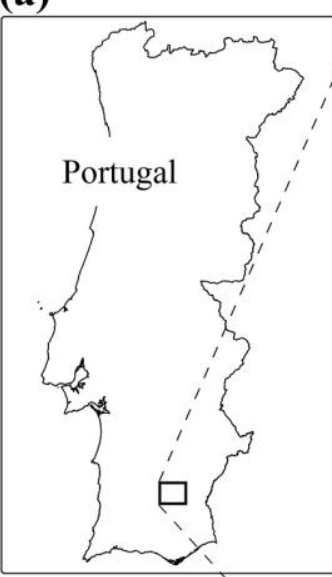

Reference data (4 AISA flightlines at $1.8 \mathrm{~m}$ g.s.d.)

Masked area

(b)

(i)

(ii)

High/medium dense shrub area different backgrounds
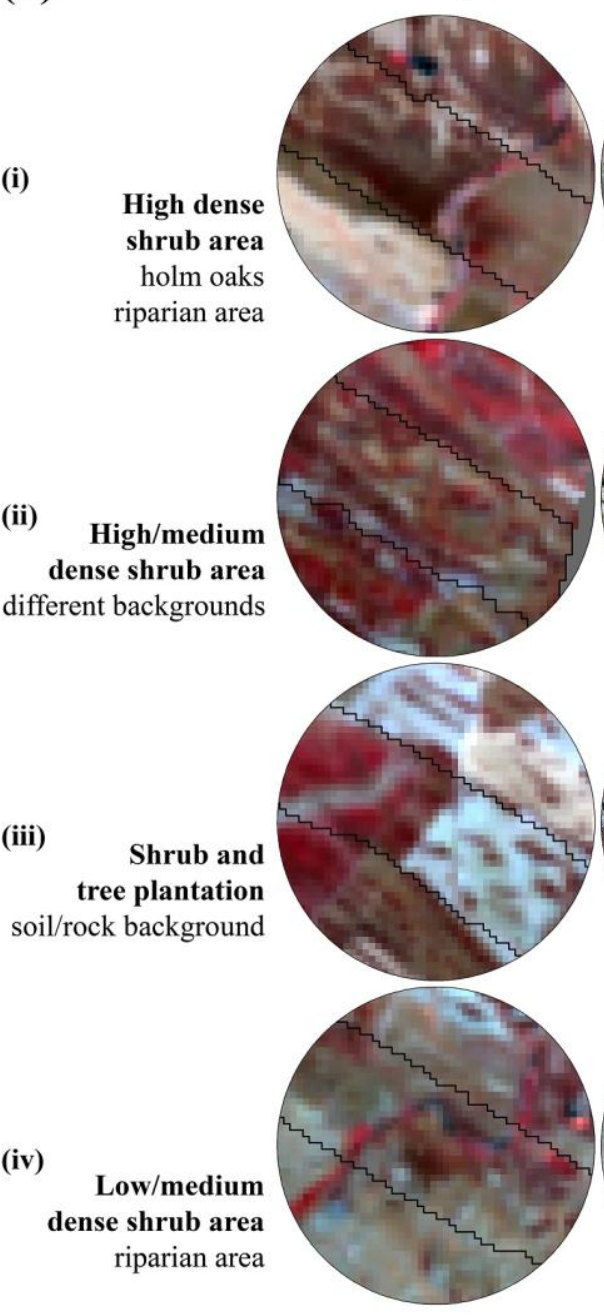

$8^{\circ} 0^{\prime} 0 " \mathrm{~W}$

$7^{\circ} 54^{\prime} 0^{\prime \prime} \mathrm{W}$

$7^{\circ} 48^{\prime} 0^{\prime \prime} \mathrm{W}$

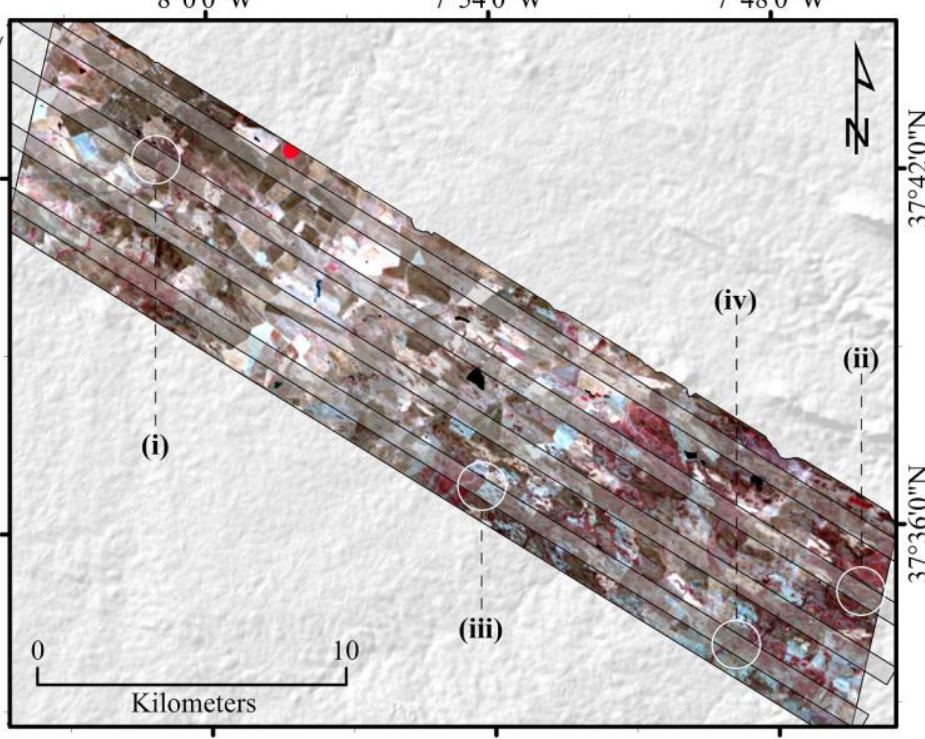

EnMAP image $(30 \mathrm{~m}) \quad$ Orthophoto $(0.5 \mathrm{~m}) \quad$ Reference map $(1.8 \mathrm{~m})$
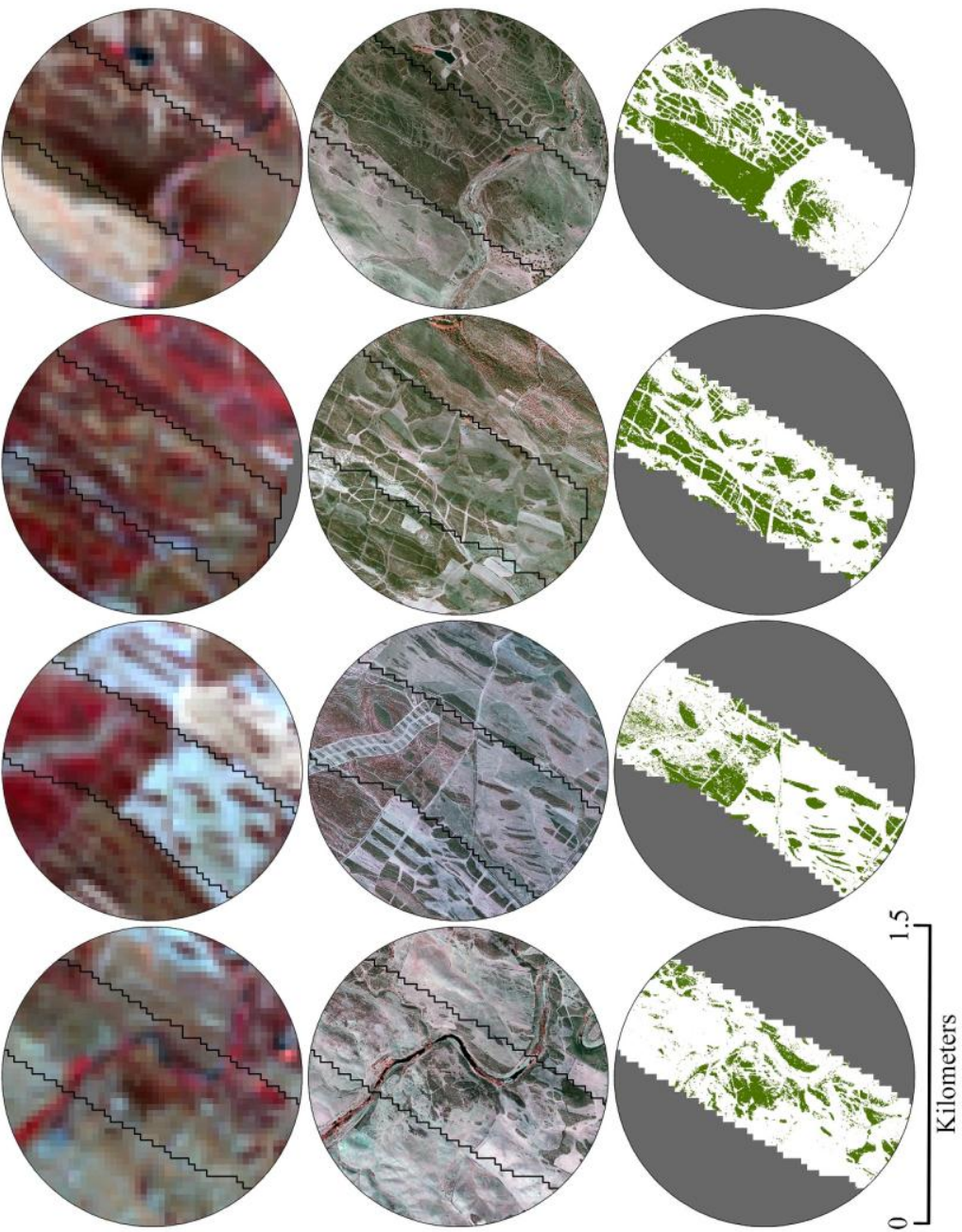

shrub

Figure 1. (a) Simulated EnMAP scene (red $=863 \mathrm{~nm}$, green $=652 \mathrm{~nm}$, blue $=548 \mathrm{~nm}$ ) covering the study area. Areas with available reference data appear semi-transparent. (b) Four selected spatial subsets are highlighted with aerial photographs (center) and a reference shrub cover map (right). 


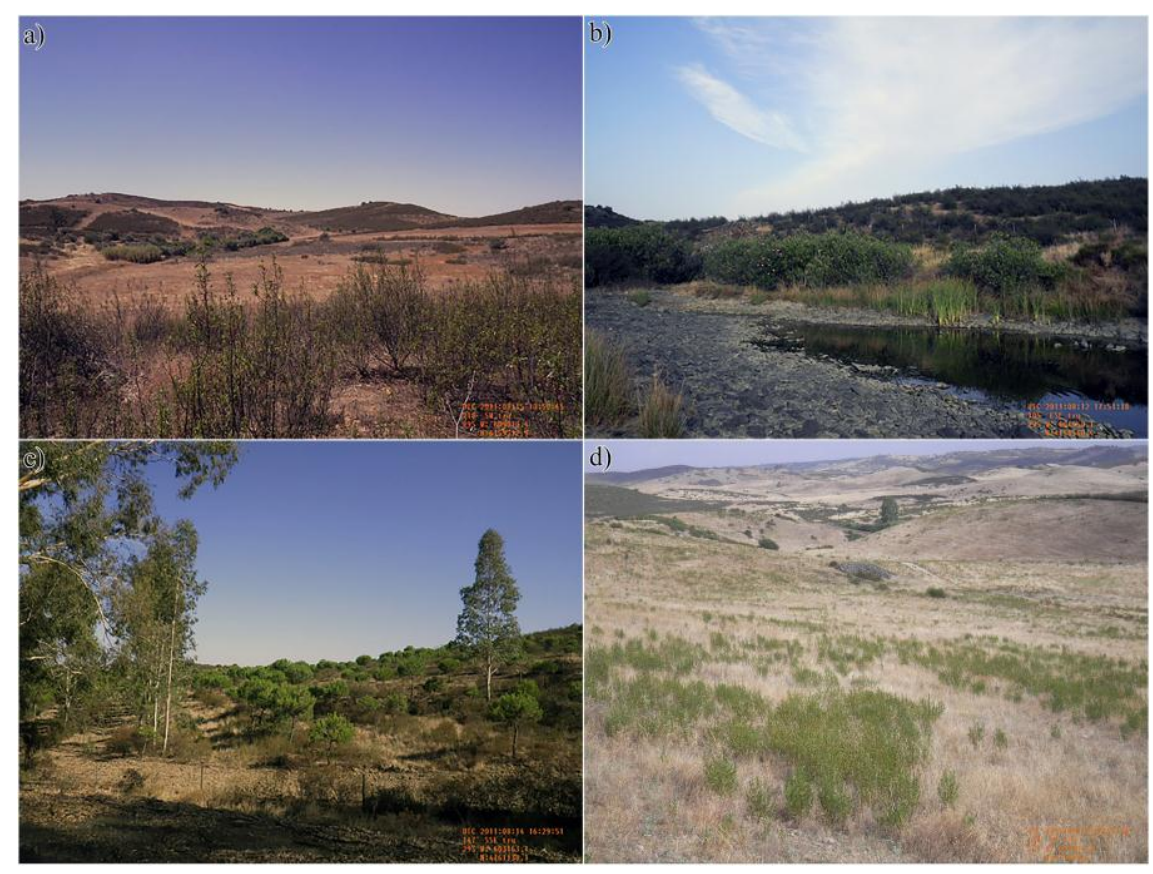

Figure 2. Photographs of shrub sites in the study area at the time of the flight campaign in August 2011. (a) Patches of Cistus spp. (medium/high dense) with dry and fallow grassland vegetation in the underlying background. (b) Riparian area with dried riverbed, Nerium oleander spp., and Phragmites vegetation and Cistus spp. patches (high dense) at the undulating background. (c) Pinus pinea plantation with Cistus spp. encroachment and Eucalyptus globulus trees. (d) Patches of scattered small shrub plants (low/medium dense) at the slope (shrub succession).

\subsection{EnMAP Data}

A simulated EnMAP scene was used for mapping shrub vegetation within the study area (Figure 1). The EnMAP scene was simulated based on four adjoined hyperspectral flight stripes acquired by the Airborne Imaging Spectrometer for Applications (AISA) at an altitude of $4500 \mathrm{~m}$ with Eagle and Hawk instruments during a flight campaign in August 2011. The AISA Eagle and Hawk datasets were radiometrically and atmospherically corrected using a radiative transfer model approach for airborne imagery with subsequent spectral polishing (Savitzky-Golay filtering), and a nadir normalization to correct for across-track illumination differences [50]. This was followed by a geometric correction, layer stacking, and mosaicking of the data [51], resulting in an AISA Eagle and Hawk reflectance product covering the entire study area in a single image mosaic with a g.s.d. of $5.4 \mathrm{~m}$. This image mosaic was subsequently used to simulate a 30 m EnMAP scene using the EnMAP end-to-end Simulation Tool (EeteS) [23,52]. EeteS was developed by the German Research Center for Geosciences (GFZ) during the preparatory activities of the EnMAP mission, and is capable of simulating EnMAP-like reflectance images (L2), taking into account a variety of instrumental and environmental configurations. The simulated EnMAP scene was corrected for noisy spectral bands and data artifacts, i.e., $0.14 \%$ of all pixels were masked. More detailed information on the flight campaign, pre-processing, and image simulation is provided in [34]. Specifications on the spectral configuration and spatial properties of the AISA and simulated EnMAP data can be found in Table 1. 
Table 1. Spectral configurations and spatial properties for EnMAP simulation input and output.

\begin{tabular}{ccc}
\hline & AISA Eagle/AISA Hawk & Simulated EnMAP \\
\hline Spectral range $(\mathrm{nm})$ & $400-970 / 970-2500$ & $420-2450$ \\
Spectral bands & $244 / 254$ & 146 (after removal of 98 bands with \\
& & low signal-to-noise ratio) \\
Spectral sampling distance $(\mathrm{nm})$ & $2.3 / 6.5$ & $6.5-10$ \\
Ground sampling distance $(\mathrm{m})$ & $5.4 / 5.4$ (after geometric correction) & 30 \\
\hline
\end{tabular}

\subsection{Training Data}

Pixels for model training were extracted from the EnMAP scene using field survey data and visual interpretation of high-resolution orthophotography data (from the "Instituto Geográfico Português", taken in 2009) ( $c f$. Figure 1b, center). A total number of 140 representative samples, reflecting all natural land cover types of the study area, were categorized into the target class shrub (44 samples) and the background class (96 samples). The target class includes pixels covered by shrub plants from the genus Cistus spp. (hereafter referred to as "shrub"). The background class includes pixels covered by the most meaningful residuary natural land cover types of the study area, such as grassland, tree, cereal, other shrub species, riparian vegetation, bare soil, exposed rock, and water. During collection of training samples for the target shrub class, we only included samples with a visually identifiable maximum natural purity with regard to the $30 \mathrm{~m}$ g.s.d. of EnMAP. In contrast, samples for the background class potentially also include mixtures of various background cover types, for example, water and trees.

\subsection{Reference Data}

Reference data for validating our shrub maps were obtained from an existing high spatial resolution land cover classification consisting of six classes (shrub (Cistus spp.), bare soil, cereal, grass, wood, and water). This land cover classification was produced in [34] with an overall accuracy (OA) of 94.2\% based on SVC and four additional AISA Eagle and Hawk flight stripes. These flight stripes were also acquired during the flight campaign in August 2011 ( $c f$. Section 2.2) at an altitude of $1500 \mathrm{~m}$, thus resulting in a lower g.s.d. of $1.8 \mathrm{~m}$ and smaller swath width when overlaid with the simulated EnMAP scene. Reference information is therefore exclusively available along four northwest- to southeast-oriented corridors within the study area ( $c f$. Figure 1a). The shrub class pixels were spatially aggregated to EnMAP pixel size, resulting in 71432 pixels (corresponding to more than $30 \%$ of the entire study area) to generate two $30 \mathrm{~m}$ resolution reference layers. The first reference layer is a discrete binary shrub/non-shrub map used to validate the hard classification, and the second reference layer is a shrub cover fraction map used to validate the fraction estimates derived from standard and adapted SVC. The reference fraction map values range from $0 \%$ to $100 \%$ shrub cover, with a large proportion of $0 \%$ shrub cover pixels.

\section{Methods and Data Analysis}

\subsection{Support Vector Classification (SVC)}

Kernel-based support vector classification from the field of machine learning has become a widely used processing technique in remote sensing research. SVC is a powerful, supervised, non-parametric, 
statistical learning technique with the ability to solve nonlinear problems, especially when adopting high-dimensional data or a small number of training samples [37,53,54]. Details on the theoretical foundation of SVC can be found in [36]. As in other kernel-based methods, the efficiency of SVC depends on the selection of adequate kernel and regularization parameters. Selecting these parameters aims at avoiding the over- or under-fitting of the model, which is commonly guaranteed using a grid search strategy combined with cross-validation of the model $[53,54]$.

We used "imageSVM" for classification [55], which is an open-source tool for support vector machine (SVM) applications with remote sensing data. imageSVM is based on the library for SVM (LIBSVM) [56] and is delivered as part of the EnMAP-Box [57]. The SVC model was initialized with the training data introduced above ( $c f$. Section 2.3). For mapping into the kernel space, we applied the Gaussian radial basis kernel function. Optimal parameter pairs for the regularization parameter $\mathrm{C}$ and kernel parameter $\gamma$ were determined by a heuristic grid search in conjunction with a three-fold cross-validation. Wide ranges of both parameters were searched to avoid the selection of pairs at the borders of the grid. The averaged F1 accuracies [58] were used as qualitative performance measures for each parameter pair during the grid search, which refers to a parameter selection optimized with regard to hard classification. The averaged F1 accuracies represent the arithmetic mean of the class-wise F1 values, which are calculated as the weighted harmonic mean of the user's $(U A)$ and producer's accuracy $(P A)$. Precisely, the class-wise $\mathrm{F} 1$ value for class $i$ is given by: $F 1_{\mathrm{i}}=2 \times U A_{\mathrm{i}} \times P A_{\mathrm{i}} /\left(U A_{\mathrm{i}}+P A_{\mathrm{i}}\right)$. Thus, being a class-dependent measure, the averaged F1 features advantages for overall measures, for example, the OA. Once the SVC is parametrized, the model output is mapped onto class probabilities by an optimized sigmoid function [41], which are then categorized into discrete classification maps by using a threshold of $50 \%$. The use of posterior probabilities is prominent as it may improve the reliability of class decisions [42]. The SVC model was applied to the EnMAP scene to derive the two-layered shrub vegetation map consisting of a hard classification (i.e., discrete shrub cover map) and a probability map of the shrub class (referred to as the sub-pixel cover fraction map). We refer to this implementation of SVC as "standard SVC".

\subsection{Adapted Support Vector Classification}

The class probabilities, as derived from standard SVC, are obtained from a model parameterization which is optimized with regard to a hard classification scheme. In order to tune the class probabilities toward a better representation of cover fractions, we tested a new strategy for parameter selection that was recently developed for combined qualitative and quantitative mapping [46]. The underlying idea of this strategy is to adapt class probabilities by using a quantitative model evaluation during parameterization. The qualitative performance measure used to evaluate each parameter pair during the grid search of standard SVC is replaced with a quantitative one. In doing so, models are not evaluated using OA, kappa coefficient, or F1 accuracy as is commonly done using discrete test samples. Instead, the difference between modeled class probabilities and mixing proportions of a set of synthetically mixed training samples is evaluated with, for example, the mean absolute error (MAE) or the root mean squared error (RMSE). The best performing model is subsequently selected for the final prediction. As synthetically mixed training data are expected to represent cover fractions [46,59], this strategy adapts probabilities to quantitative mapping.

The synthetically mixed data considered for model selection were calculated by mixing each training sample from the target class with every available background pixel [46,59]. To represent cover fractions 
we assumed simple linear mixing between the spectra, which applies to many real-world scenarios [60]. We used mixing proportions in $20 \%$ steps. The synthetic dataset, therefore, consists of synthetic spectra representing mixtures between the target class (shrub) and the background class (i.e., all remaining cover types). Associated mixing fractions are: 20/80; 40/60; 60/40; 80/20 (proportion target class/proportion background class in $\%)$. This resulted in a set of original pure class spectra $(44+96=140)$, synthetically mixed spectra $(44 \times 96 \times 4=16,896)$, and related mixing proportions from $0 \%$ to $100 \%$ of the respective target class.

We used an adapted version of imageSVM for classification based on the introduced quantitative model selection. The general framework was kept constant to standard SVC, i.e., the same training input and model settings were adopted to guarantee a fair comparison of the methods. Nevertheless, according to this strategy, we chose the MAE as the quantitative performance measure during the grid search, together with 17,036 spectra and mixing proportions for model evaluation. By applying the adapted SVC model to the EnMAP scene, we obtained another two-layered shrub vegetation map consisting of a hard classification (i.e., discrete shrub cover map) and a probability map of the shrub class (representing a sub-pixel cover fraction map). We refer to this implementation of SVC as "adapted SVC".

\subsection{Validation}

The accuracies of these shrub vegetation maps produced from both standard and adapted SVC were evaluated using the independent reference data at EnMAP pixel scale. The discrete shrub cover maps were compared to the discrete reference information and statistically evaluated for their averaged F1 accuracy. The shrub cover fraction maps were compared to the reference fractions and evaluated using the MAE, RMSE, and goodness of fit statistics ( $\mathrm{R}^{2}$ values). All statistics were calculated for the entire reference area and for the four selected spatial subsets. To account for the strongly skewed shrub cover distribution ( $c f$. Section 2.4), we considered an unbiased equalized random sample from the reference data of 731 observations for each reference decile for the entire study area. In this way, we derived 7310 validation pixels, which ensured a more realistic evaluation along the full range of fractions. To account for random effects during sampling, we used mean values over 200 iterations. For evaluating the four spatial subsets, we used all available reference samples within the respective subset (889 validation pixels in (i), 830 in (ii), 858 in (iii), and 891 in (iv)). For better visualization of the high number of validation pixels, a boxplot of each reference decile is presented.

\section{Results}

\subsection{Assessment of Discrete Shrub Cover Maps}

The discrete shrub cover maps derived by standard and adapted SVC (Figure 3a) illustrate similar spatial patterns with a dominance of shrub cover in the southeastern part of the study area. Adapted SVC leads to an improved F1 accuracy of $81.3 \%$ compared to standard SVC with $72.4 \%$ (cf. Table 2a). Exploring mapping patterns and accuracies at the local scale (selected subsets) provides a more detailed insight into the quality of the discrete shrub cover map for different regions within the study area. The visual inspection of the four subset regions more clearly reveals spatial patterns of shrub vegetation, which are well reproduced by both SVC variants ( $c f$. Figure $3 b$ ). Adapted SVC results in more shrub 
pixels. This is clearly visible in the classification subsets, where, in comparison with standard SVC, for (i, ii) $20 \%$ to $30 \%$ more shrub pixels are present, for (iii) nearly double the number are present, and in (iv) almost three times the number of pixels are classified as shrub ( $c f$. Figure $3 b$ ). The classification results show that shrub vegetation is separated from different background cover types, such as herbaceous vegetation, grass, and soil (i-iv), trees (i, iii), and riparian land cover types (i, iv), which is also expressed by the F1 accuracies ( $c f$. Table 2b). In (iv), accuracies are distinctly lower for adapted SVC, which is mainly related to errors in the northwestern part of the reference area.

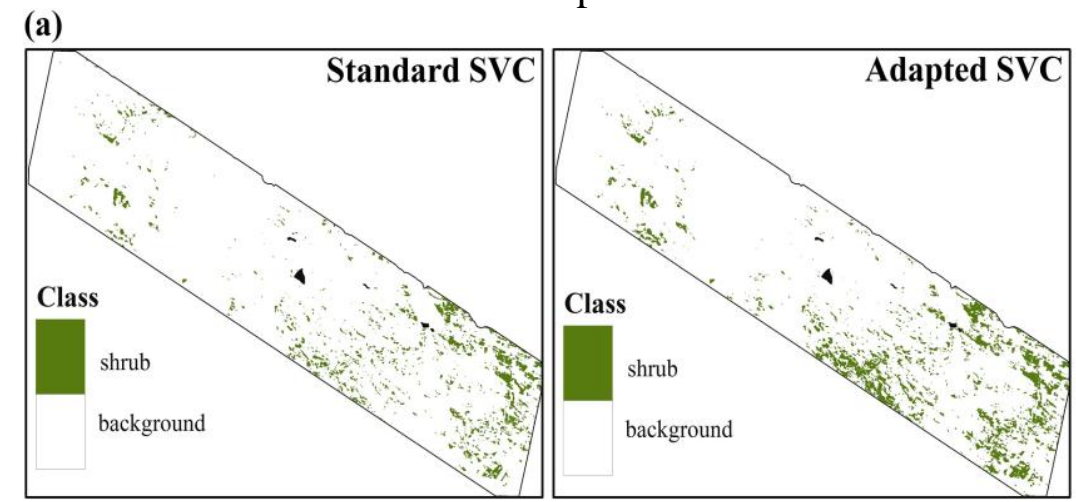

(b)

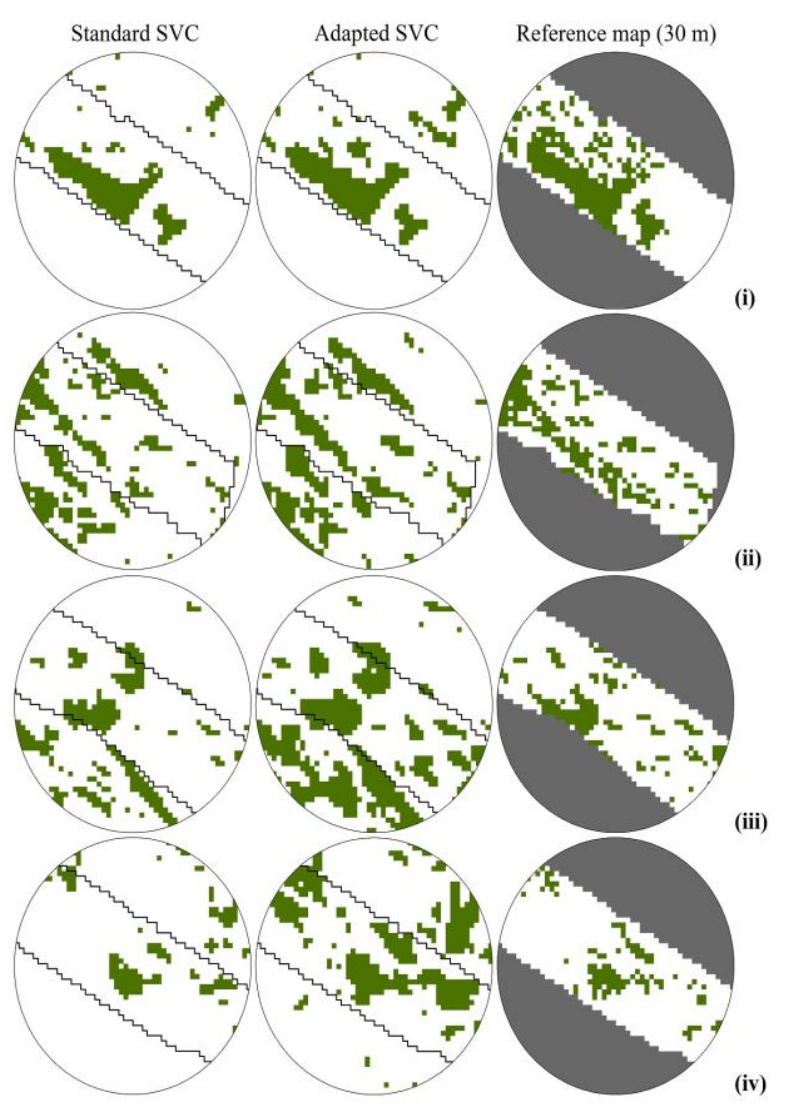

Figure 3. Discrete shrub cover maps derived from standard SVC and adapted SVC (a) for the entire study area and (b) for selected subsets. For information on coordinates and orientation, the reader is referred to Figure 1. 
Table 2. Averaged F1 accuracies of the discrete shrub cover maps derived from standard SVC (left) and adapted SVC (right) (a) for the entire study area and (b) for selected subsets. Statistics for the entire study area are derived from mean values of 200 iterations for an equalized random sample of 731 observations for each decile ( $c f$. Section 3.3). Higher accuracy values are highlighted.

\begin{tabular}{llccc}
\hline & & Standard SVC & Adapted SVC & \\
\hline (a) & F1(\%) & 72.37 & $\mathbf{8 1 . 3 3}$ & \\
\hline (b) & F1(\%) & 71.15 & $\mathbf{7 5 . 6 5}$ & (i) \\
& & 64.41 & $\mathbf{6 7 . 5 0}$ & (ii) \\
& & 62.88 & $\mathbf{6 3 . 8 3}$ & (iii) \\
& & $\mathbf{5 5 . 1 0}$ & 48.17 & (iv) \\
\hline
\end{tabular}

\subsection{Assessment of Shrub Cover Fraction Maps}

The shrub cover fraction maps derived by standard and adapted SVC clearly reveal the landscape patterns of gradual transitions in shrub vegetation, with increasing shrub abundance toward the southeastern part of the study area ( $c f$. Figure 4a). Quantitative accuracies of the shrub cover fraction maps obtained from standard and adapted SVC are shown in Table 3. Adapted SVC performs better and improves shrub cover fraction estimates, with RMSE decreasing from $29.5 \%$ to $23.2 \%$, i.e., a relative decrease of RMSE by $21.4 \%$; the $\mathrm{R}^{2}$ value increases to $53.1 \%$ ( $c f$. Table $3 \mathrm{a}$ ), based on the validation with an equalized random sample ( $c f$. Section 3.3). This is also shown by the boxplots (Figure 5a) for estimated fractions per reference decile. These illustrate an underestimation of shrub cover fractions derived from standard SVC, expressed by median values permanently below the 1:1 line. In contrast, the estimations obtained from adapted SVC uniformly scatter around the 1:1 line, expressed by median values better matching the reference values and a smaller variance ( $c f$. Figure $5 a)$.

Table 3. Accuracies of shrub cover fraction maps derived from standard SVC and adapted SVC (a) for the entire study area and (b) for selected subsets. Statistics for the entire study area are derived from mean values of 200 iterations for an equalized random sample of 731 observations for each decile ( $c f$. Section 3.3). Higher accuracy values are highlighted.

\begin{tabular}{|c|c|c|c|c|}
\hline & & Standard SVC & Adapted SVC & \\
\hline \multirow[t]{3}{*}{ (a) } & $\operatorname{MAE}(\%)$ & 17.10 & 16.28 & \\
\hline & $\operatorname{RMSE}(\%)$ & 29.52 & 23.20 & \\
\hline & $\mathrm{R}^{2}(\%)$ & 47.30 & 53.12 & \\
\hline \multirow[t]{12}{*}{ (b) } & $\operatorname{MAE}(\%)$ & 12.95 & 11.14 & \\
\hline & $\operatorname{RMSE}(\%)$ & 19.56 & 16.68 & (i) \\
\hline & $\mathrm{R}^{2}(\%)$ & 76.00 & 79.82 & \\
\hline & $\operatorname{MAE}(\%)$ & 14.54 & 12.77 & \\
\hline & $\operatorname{RMSE}(\%)$ & 20.24 & 17.54 & (ii) \\
\hline & $\mathrm{R}^{2}(\%)$ & 61.05 & 66.88 & \\
\hline & $\operatorname{MAE}(\%)$ & 14.12 & 11.32 & \\
\hline & $\operatorname{RMSE}(\%)$ & 21.61 & 20.33 & (iii) \\
\hline & $\mathrm{R}^{2}(\%)$ & 50.33 & 61.98 & \\
\hline & $\operatorname{MAE}(\%)$ & 11.79 & 13.25 & \\
\hline & $\operatorname{RMSE}(\%)$ & 18.32 & 30.25 & (iv) \\
\hline & $\mathrm{R}^{2}(\%)$ & 49.32 & 42.91 & \\
\hline
\end{tabular}


Inspecting statistics on a local scale (Table 3b), this contrast is particularly obvious in (iii), where the $\mathrm{R}^{2}$ value increased from $50.3 \%$ to $62.0 \%$ and MAE decreased from $14.1 \%$ to $11.3 \%$, with distinctly lower errors in the eastern parts of the reference area ( $c f$. Figure 4b). Again, in (iv), accuracies are distinctly lower for adapted SVC ( $c f$. Table $3 b$ ), with a high proportion of overestimation at low to medium dense reference fractions ( $c f$. Figure $5 b$ (iv)). In general, however, inspecting the decile-wise boxplots on the local scale (Figure 5b), adapted SVC slightly underestimates dense shrub areas (i, ii) with maximal values close to $90 \%$. In contrast to this, standard SVC underestimates intermediate fractions. Estimations slightly increase and then jump to higher fractions, expressed through abrupt increasing median values at about $80 \%$ ( $c f$. Figure $5 \mathrm{~b}$ (i, iii)). This translates into a more class-pronounced appearance of the shrub cover fraction maps. In contrast to this, adapted SVC reveals a more gradual transition in shrub vegetation.

(a)

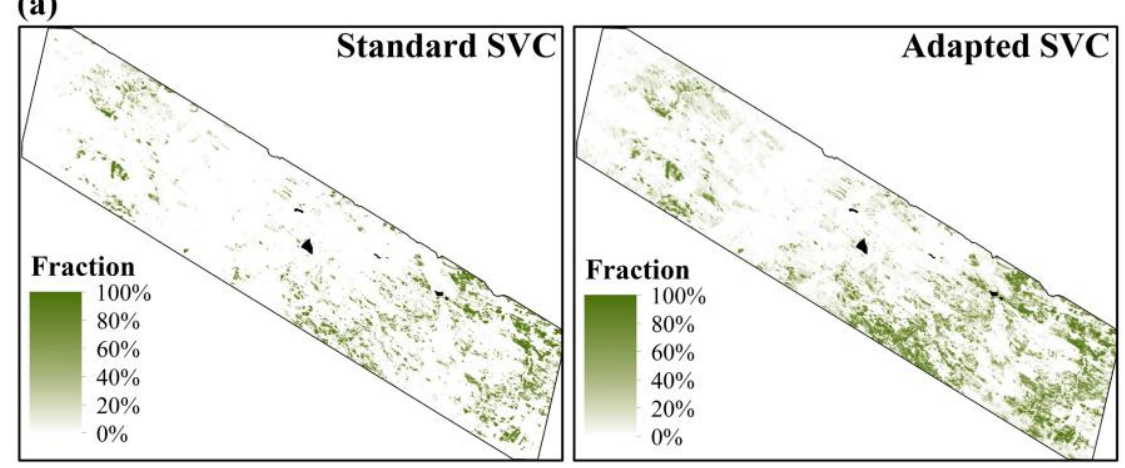

(b)

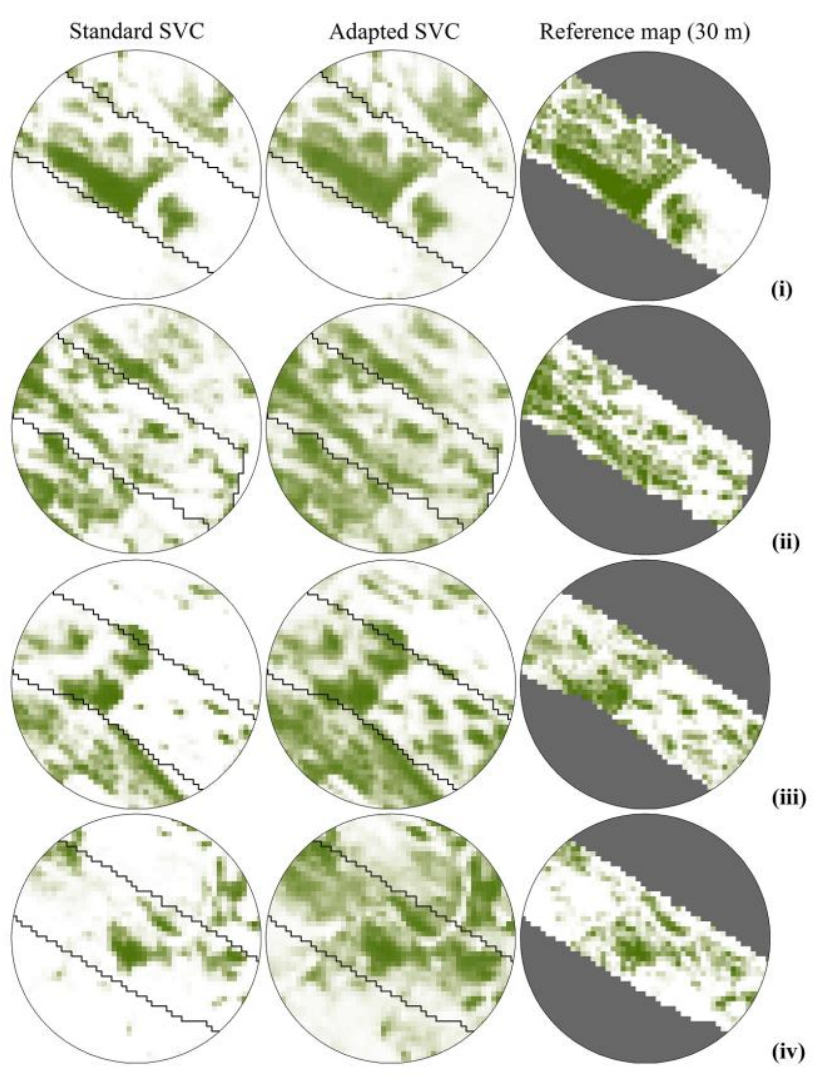

Figure 4. Shrub cover fraction maps derived from standard SVC and adapted SVC (a) for the entire study area and (b) for selected subsets. For information on coordinates and orientation, the reader is referred to Figure 1. 
(a)

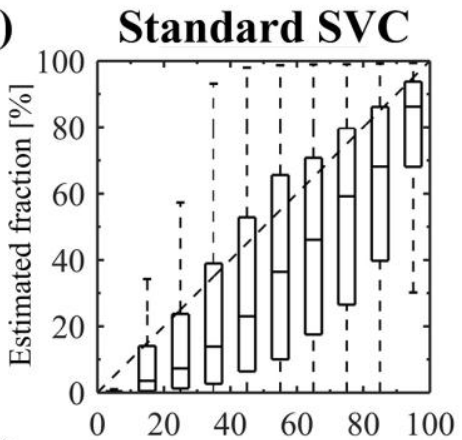

(b)
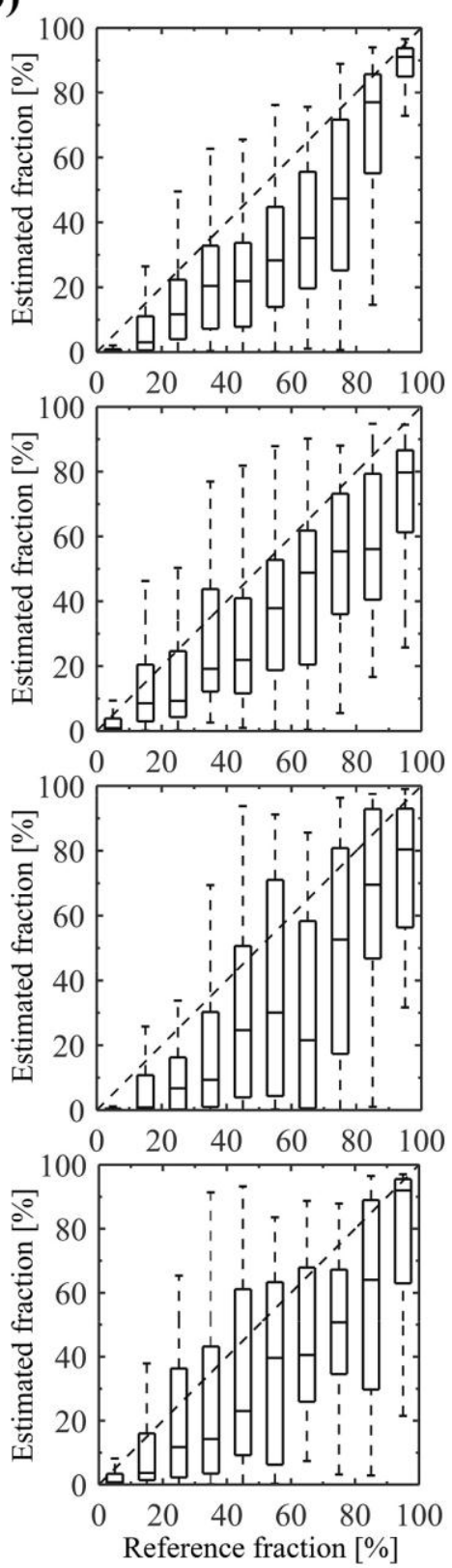

Adapted SVC
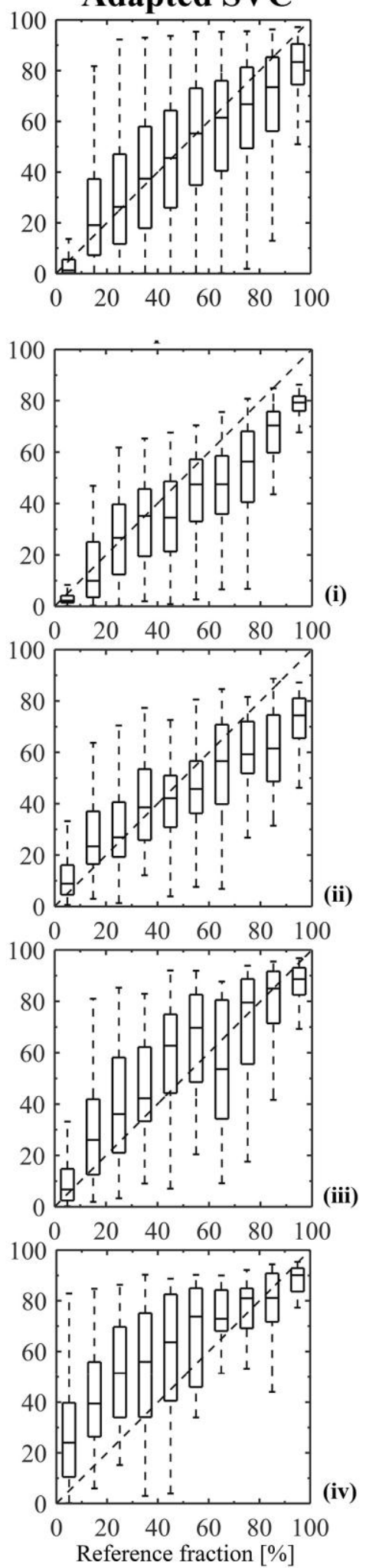

Figure 5. Decil-wise boxplot diagrams of the shrub cover fraction maps derived from standard SVC (left) and adapted SVC (right) (a) for the entire study area and (b) for selected subsets. For each box of the boxplots, the central mark is the median, the edges of the box are the 25th and 75th percentiles, and the whiskers extend to the outliers [61]. Perfect prediction is highlighted with a dashed 1:1 line. 


\section{Discussion}

In this paper, we evaluated the use of an adapted SVC approach, which is capable of producing discrete classification maps of the dominant land cover and simultaneously generates land cover fraction maps to better represent gradual landscape transitions. The concept behind this strategy is the quantitative model evaluation during parameterization to adjust class probabilities to the prediction of cover fractions. The approach was previously tested using library spectra [46] and is now applied to simulated EnMAP data covering a landscape gradient of shrub encroachment in a semi-arid, natural environment in Portugal. We considered the binary, two-class problem of shrub vegetation vs. background. While the target shrub class only included areas covered by shrub plants from a single genus (Cistus spp.) and is thus spectrally very homogeneous, the background class is highly heterogeneous as it consisted of most remaining natural land cover types presented in the scenery, including other shrub species (e.g., Nerium oleander), other vegetation types (e.g., grassland, trees, riverine vegetation), dried riverbed sediments, bare soil, rocks, and water. The resulting spectral complexity, similarities, and multi-modal distributions made the classification task very challenging.

Our first objective was to evaluate the quality of the discrete shrub classification maps derived from standard SVC and adapted SVC, because maintaining the high discriminative power of the SVC was a precondition to evaluate the adapted SVC. Both SVC approaches show their strength to sharply separate the target shrub cover from different background cover types ( $c f$. Figure 3 ). This also applies for complex land cover compositions where shrub vegetation is present underneath trees in tree plantations ( $c f$. Figure $3 \mathrm{~b}$ (iii)). This is in line with most studies showing that SVCs are well suited to deal with high data dimensionality, spectral complexity, similarity, and variability [39,62]. Looking at the F1 accuracies for the entire study area (Table $2 \mathrm{a})$, adapted SVC is clearly superior to standard SVC $(\Delta \mathrm{F} 1=+9.0 \%)$. To some extent, this relates to the insufficient discrimination of shrub pixels by standard SVC, resulting in a too conservative estimation, i.e., a larger omission error due to the underestimation of shrub cover. This effect is more clearly visible in the classification subsets (Figure $3 b$ ). Thus, the suggested adaptation of the SVC parameterization appears to improve the separation of the target and background class. By including synthetically mixed spectral information that is closer to the actual class boundaries than pure spectra, the high share of mixed pixels is better represented in the model process. This is in line with the argumentation in [63], where the authors have also intentionally used mixed pixels. Our approach, however, is solely based on pure training pixels and synthetically mixed spectral information calculated thereof, and as such imposes no extra work on the user.

Our second objective was to evaluate the accuracy of fraction maps derived from the probability output of standard and adapted SVC. Effective assignment of continuous shrub fractions is illustrated in Figure 4, where the patterns of shrub fraction distribution are in good agreement with the reference. For a realistic estimate of cover fractions, adapted SVC also proves to be the superior approach when considering the entire study area, expressed by decreased errors $(\triangle \mathrm{MAE}=-0.8 \%, \Delta \mathrm{RMSE}=-6.3 \%$ ) and an increased goodness of fit $\left(\Delta \mathrm{R}^{2}=+5.8 \%\right)$. These summarizing statistics can be confirmed for three of the four spatial subsets, where they are sometimes even more positive ( $c f$. Table 3). Particularly, the high improvement in RMSE indicates that large errors, which are more pronounced by using RMSE than MAE, are reduced by adapted SVC. Similar to the discrete classification case, the poorer performance of standard SVC relates, to some extent, to underestimations in shrub cover fractions. In contrast, the 
adapted SVC parameterization with synthetic mixtures positively affects quantitative predictions due to a better fitting of continuous model outputs, i.e., the class probabilities, to land cover fractions. As shown in [45], the standard parameterization often results in many parameter pairs of identical or very similar accuracy in the internal grid search and cross-validation. This is not the case for the adapted SVC where the parameter selection appears distinct. Our findings are in line with [46], and here are, for the first time, demonstrated on actual image data with complex mixing structures.

The comparison between standard and adapted SVC clearly shows the improvement in shrub map quality through little changes in the parameterization process of the classifier. Yet, with an averaged F1 accuracy of $81.3 \%$ and an RMSE of $23.2 \%$, shrub map accuracies are not as high as commonly reported for mapping assessments in other ecosystems (e.g., forest). On the one hand, this relates to the heterogeneous patterns and gradual transition of shrub vegetation ( $c f$. photos in Figure 2), especially when quantifying sparse vegetation types at a $30 \mathrm{~m}$ EnMAP scale. The challenges and limitations when mapping sparse vegetation in arid and semiarid environments were broadly discussed [64-67]: uncertainties are mainly referred to as the presence of indeterminate vegetation types, the low spectral contrast, the large radiation from bright soils and rocks overwhelming the lower vegetation signal, and the spectral coupling effect. On the other hand, the accuracies achieved with class-independent, i.e., overall, measures (MAE, RMSE, and $\mathrm{R}^{2}$ ) strongly depend on the sampling strategy used for validation. We selected a representative sampling strategy that accounts for the strongly skewed shrub cover distribution of the reference fractions, i.e., we used an equalized random sample for each reference fraction decile and in this way emphasize the especially challenging central deciles. When using a spatially representative random sample and taking into account the high share of reference pixels with low cover fractions, the RMSE is at $14.4 \%$ instead of $23.2 \%$ and is closer to values in other successful studies.

Despite the overall improved performance, we also revealed large discrepancies between the fraction maps derived from adapted SVC and the reference map. We identified areas (e.g., Figure 4b (iv)) where our estimates revealed around $30 \%$ to $50 \%$ cover fractions, whereas the reference indicates no shrub cover. These problematic areas were also inspected by photographs, where we recognized the existence of early shrub succession. At this point, limitations of the reference data become obvious [34]. The classification of a high spatial resolution image of $1.8 \mathrm{~m}$ with subsequent spatial aggregation to $30 \mathrm{~m}$ was a straightforward and practicable procedure to create a large reference dataset. It allowed for an extensive accuracy assessment and detailed analysis of errors. However, the mixing of different land cover types at those high spatial resolutions will amplify discrepancies between the aggregated classification map and the reality. Validation becomes particularly problematic when considering wide areas of an early successional stage with low to medium dense shrub cover, as illustrated in Figure $2 \mathrm{~d}$. Here, the reference map may show large areas of non-shrub pixels which are then aggregated to $0 \%$ shrub cover, although the full area may have homogenous sparse shrub cover. Indeed, discrepancies between results from adapted SVC and the reference map were highest for areas with scattered small shrub plants and seedlings, and the resulting poor accuracies can be explained in favor of our approach ( $c f$. Table $3 \mathrm{~b}$ (iv), Figure 5b (iv)). Data analysis shows that our approach better reflects the reality as low to medium dense shrub pixels were imposingly recognized, which highlights the sensitivity and applicability of this method to areas of shrub succession. Thus, statistic measures indicate discrepancies between reference data and reality and underestimate accuracies, which additionally should increase actual accuracies of adapted SVC and further qualify adapted SVC over standard SVC. In a similar way, 
the slight underestimation of dense shrub cover may be explained, where the reference map shows an aggregated $90 \%$ to $100 \%$ cover, because of the hard classification at $1.8 \mathrm{~m}$, while the adapted SVC recognizes the certain share of non-shrub cover within such areas.

Mapping of shrub cover fractions based on class probabilities from adapted SVC yielded promising results. Therefore, the approach marks a step in bridging the often dichotomous distinction between qualitative and quantitative analysis. Beside the improved accuracy of hard classification, the quantitative results prove comparable to those from purely quantitative approaches. In [34], the authors tested the performance of three regression methods, including support vector regression (SVR), random forest regression, and partial least squares regression, to estimate shrub cover fractions, based on the same EnMAP scene and reference map. They achieved a maximum accuracy of $12 \%$ RMSE based on a spatially representative random sample, which is in the range of the results presented here, where the spatially representative sampling leads to an RMSE of 14.4\%. The beneficial effect of adapted SVC is that it only requires pure spectra for training which can be labeled from the image, whereas regression approaches as in [34] require spectra from mixed pixels together with accurate information on mixing proportions, which is usually not available; it mostly depends on spatially aggregated training information derived from higher-resolution reference maps, as it was the case in the study by [34]. The proposed adapted SVC approach is hence more flexible. This finding, in combination with the convincing accuracies, strengthens the application potential of the adapted classification approach for combined qualitative and quantitative analysis.

\section{Conclusion}

The classification of land cover is a widely used procedure to analyze earth observation data, leading to discrete results, which are easy to interpret and sufficient for many subsequent applications. However, they do not account for spectral mixing between different land cover types and often fail to realistically represent environments characterized by gradual transitions, which are typical for natural environments at the spatial scale of EnMAP data. It is therefore reasonable to offer easy-to-use classification approaches which accurately map the dominant land cover and simultaneously generate sub-pixel cover fraction estimates. In this paper, we propose a new, adapted approach for SVC parameter selection, where probability outputs are adjusted to represent cover fractions using synthetically mixed data, to extend a hard classification with information on shrub cover fractions. While synthetic mixtures were previously incorporated into the parameterization process of machine learning-based classification with success [46], an application on actual image data with complex mixtures was still lacking. Therefore, we used simulated EnMAP data to map shrub vegetation along a landscape gradient of shrub encroachment in southern Portugal. We derived a two-layered shrub vegetation map of hard classification and fraction representation of the shrub cover. We compared the performance of adapted SVC parameterization with the standard one. By using an unbiased equalized random sampling validation strategy, our results show that shrub class probabilities from adapted SVC more accurately represent shrub cover fractions (MAE/RMSE of 16.3\%/23.2\%) compared to standard SVC (17.1\%/29.5\%). Simultaneously, the discrete classification output was considerably improved (averaged $\mathrm{F} 1$ accuracies increased from $72.4 \%$ to $81.3 \%$ ).

Based on our findings, the integration of synthetic mixtures into the parameterization of machine learning-based classification in general and in SVC parameterization in particular can be recommended for deriving improved qualitative and quantitative gradient maps, bridging the often dichotomous 
distinction between qualitative and quantitative analysis. The mapping of shrub cover fractions with class probabilities from adapted SVC yields promising results, which are competitive with state-of-the-art regression models, with the great advantage not requiring quantitative input. Therefore, we see benefits in this adapted parameterization framework to perform combined qualitative and quantitative mapping due to its efficient and user-friendly applicability. It is certainly worth being tested in other approaches and on different datasets in future studies. The approach is also scheduled to be implemented in future EnMAP-Box versions [57]. We see a high relevance for future EnMAP data together with advanced machine learning-based classification approaches for the accurate mapping of natural ecosystems on a frequent and area-wide basis, with unique opportunities to derive improved and realistic descriptions of gradual transitions. These data products can likely support a better understanding of land change processes and sustainable management strategies maintaining natural ecosystems to ensure the conservation of biodiversity and provision of essential ecosystem services.

\section{Acknowledgments}

This research is part of the EnMAP Core Science Team (ECST), which was funded by the German Aerospace Centre (DLR)-Project Management Agency, granted by the Ministry of Economics and Technology (BMWi; grant nr. 50EE0949). This work was also partly funded by the European Facility for Airborne Research (EUFAR) with the HyMedEcos-Gradients project (EUFAR 11-04) with support from the Airborne Research and Survey Facility (ARSF), Geodetic Equipment Facility (GEF) and Field Spectroscopy Facility (FSF) of the UK's Natural Environmental Research Council (NERC). The authors would also like to thank Karl Segl from the German Research Center for Geosciences (GFZ), for EnMAP data simulation; and are grateful to the valuable communication with Maren-Wanda Wolf (Department of Mathematics, Freie Universität Berlin), and Andreas Rabe (Geography Department, Humboldt-Universität zu Berlin). Finally, the authors would like to thank the anonymous reviewers for the valuable comments and suggestions, which further improved the manuscript.

\section{Author Contributions}

Stefan Suess designed this research, performed the analysis, analyzed the data, and wrote the manuscript. The co-authors of this manuscript considerably contributed at all phases of the investigation. Sebastian van der Linden, Akpona Okujeni, and Pedro J. Leitão supervised this research, contributed to the research design, and to the manuscript writing. Marcel Schwieder provided data, and contributed to the manuscript writing. Patrick Hostert contributed to the manuscript writing.

\section{Conflicts of Interest}

The authors declare no conflict of interest.

\section{References}

1. Millennium Ecosystem Assesement. Millennium Ecosystem Assessment Synthesis Reports; Island Press: Washington, DC, USA, 2006. 
2. Fonseca, F.; de Figueiredo, T.; Bompastor Ramos, M.A. Carbon storage in the Mediterranean upland shrub communities of Montesinho Natural Park, northeast of Portugal. Agrofor. Syst. 2012, $86,463-475$.

3. Shoshany, M.; Karnibad, L. Mapping shrubland biomass along Mediterranean climatic gradients: The synergy of rainfall-based and NDVI-based models. Int. J. Remote Sens. 2011, 32, 9497-9508.

4. Vogiatzakis, I.N.; Mannion, A.M.; Griffiths, G.H. Mediterranean ecosystems: Problems and tools for conservation. Prog. Phys. Geogr. 2006, 30, 175-200.

5. Moreira, F.; Russo, D. Modelling the impact of agricultural abandonment and wildfires on vertebrate diversity in Mediterranean Europe. Landsc. Ecol. 2007, 22, 1461-1476.

6. Van Auken, O.W. Causes and consequences of woody plant encroachment into western North American grasslands. J. Environ. Manage. 2009, 90, 2931-2942.

7. Eldridge, D.J.; Bowker, M.A.; Maestre, F.T.; Roger, E.; Reynolds, J.F.; Whitford, W.G. Impacts of shrub encroachment on ecosystem structure and functioning: Towards a global synthesis. Ecol. Lett. 2011, 14, 709-722.

8. Graetz, R.D. Remote sensing of terrestrial ecosystem structure: An ecologist's pragmatic view. In Ecological Studies: Remote Sensing of Biosphere Functioning; Hobbs, R.J., Mooney, H.A., Eds.; Springer Science \& Business Media: Berlin, Germany, 1990; pp. 5-30.

9. Shoshany, M. Satellite remote sensing of natural Mediterranean vegetation: A review within an ecological context. Prog. Phys. Geogr. 2000, 24, 53-78.

10. Hamada, Y.; Stow, D.A.; Roberts, D.A. Estimating life-form cover fractions in California sage scrub communities using multispectral remote sensing. Remote Sens. Environ. 2011, 115, 3056-3068.

11. Selkowitz, D.J. A comparison of multi-spectral, multi-angular, and multi-temporal remote sensing datasets for fractional shrub canopy mapping in Arctic Alaska. Remote Sens. Environ. 2010, 114, $1338-1352$.

12. Silvan-Cardenas, J.L.; Wang, L. Retrieval of subpixel Tamarix canopy cover from Landsat data along the Forgotten River using linear and nonlinear spectral mixture models. Remote Sens. Environ. 2010, 114, 1777-1790.

13. Laliberte, A.S.; Rango, A.; Havstad, K.M.; Paris, J.F.; Beck, R.F.; McNeely, R.; Gonzalez, A.L. Object-oriented image analysis for mapping shrub encroachment from 1937 to 2003 in southern New Mexico. Remote Sens. Environ. 2004, 93, 198-210.

14. McGlynn, I.O.; Okin, G.S. Characterization of shrub distribution using high spatial resolution remote sensing: Ecosystem implications for a former Chihuahuan Desert grassland. Remote Sens. Environ. 2006, 101, 554-566.

15. Lawrence, R.L.; Wood, S.D.; Sheley, R.L. Mapping invasive plants using hyperspectral imagery and Breiman Cutler classifications (randomForest). Remote Sens. Environ. 2006, 100, 356-362.

16. Jafari, R.; Lewis, M.M. Arid land characterisation with EO-1 Hyperion hyperspectral data. Int. J. Appl. Earth Obs. Geoinf. 2012, 19, 298-307.

17. Hamada, Y.; Stow, D.A.; Coulter, L.L.; Jafolla, J.C.; Hendricks, L.W. Detecting Tamarisk species (Tamarix spp.) in riparian habitats of Southern California using high spatial resolution hyperspectral imagery. Remote Sens. Environ. 2007, 109, 237-248.

18. Miao, X.; Patil, R.; Heaton, J.S.; Tracy, R.C. Detection and classification of invasive saltcedar through high spatial resolution airborne hyperspectral imagery. Int. J. Remote Sens. 2011, 32, 2131-2150. 
19. Thorp, K.R.; French, A.N.; Rango, A. Effect of image spatial and spectral characteristics on mapping semi-arid rangeland vegetation using multiple endmember spectral mixture analysis (MESMA). Remote Sens. Environ. 2013, 132, 120-130.

20. Chopping, M.; Su, L.; Laliberte, A.; Rango, A.; Peters, D.P.C.; Kollikkathara, N. Mapping shrub abundance in desert grasslands using geometric-optical modeling and multi-angle remote sensing with CHRIS/PROBA. Remote Sens. Environ. 2006, 104, 62-73.

21. Stagakis, S.; Markos, N.; Sykioti, O.; Kyparissis, A. Monitoring canopy biophysical and biochemical parameters in ecosystem scale using satellite hyperspectral imagery: An application on a Phlomis fruticosa Mediterranean ecosystem using multiangular CHRIS/PROBA observations. Remote Sens. Environ. 2010, 114, 977-994.

22. Blanco, P.D.; del Valle, H.F.; Bouza, P.J.; Metternicht, G.I.; Hardtke, L.A. Ecological site classification of semiarid rangelands: Synergistic use of Landsat and Hyperion imagery. Int. J. Appl. Earth Obs. Geoinf. 2014, 29, 11-21.

23. Guanter, L.; Kaufmann, H.; Segl, K.; Foerster, S.; Rogass, C.; Chabrillat, S.; Kuester, T.; Hollstein, A.; Rossner, G.; Chlebek, C.; et al. The EnMAP spaceborne imaging spectroscopy mission for earth observation. Remote Sens. 2015, 7, 8830.

24. Olsson, A.D.; Morisette, J.T. Comparison of simulated HyspIRI with two multispectral sensors for invasive species mapping. Photogramm. Eng. Remote Sens. 2014, 80, 217-227.

25. Leitão, P.J.; Schwieder, M.; Suess, S.; Okujeni, A.; Galvão, L.S.; van der Linden, S. Hostert, P. Monitoring natural ecosystem and ecological gradients: Perspectives with EnMAP. Remote Sens. 2015, Accepted.

26. Rosenqvist, A.; Milne, A.; Lucas, R.; Imhoff, M.; Dobson, C. A review of remote sensing technology in support of the Kyoto Protocol. Environ. Sci. Policy 2003, 6, 441-455.

27. Kaufmann, H.; Segl, K.; Itzerott, S.; Bach, H.; Wagner, A.; Hill, J.; Heim, B.; Oppermann, K.; Heldens, W.; Stein, E.; et al. Hyperspectral Algorithms: Report of the Frame of EnMAP Preparation Activities; German Research Center for Geosciences (GFZ): Potsdam, Germany, 2008.

28. Keshava, N.; Mustard, J.F. Spectral unmixing. IEEE Signal Process. Mag. 2002, 19, 44-57.

29. Shoshany, M.; Svoray, T. Multidate adaptive unmixing and its application to analysis of ecosystem transitions along a climatic gradient. Remote Sens. Environ. 2002, 82, 5-20.

30. Foody, G.M. A fuzzy-sets approach to the representation of vegetation continua from remotely sensed data-An example from lowland heath. Photogramm. Eng. Remote Sens. 1992, 58, 221-225.

31. Pan, Y.Z.; Hu, T.G.; Zhu, X.F.; Zhang, J.S.; Wang, X.D. Mapping cropland distributions using a hard and soft classification model. IEEE Trans. Geosci. Remote Sens. 2012, 50, 4301-4312.

32. Manslow, J.; Brown, M.; Nixon, M. On the Probabilistic Interpretation of Area Based Fuzzy Land Cover Mixing Proportions; Springer-Verlag: Berlin, Germany, 2000.

33. Roberts, D.A.; Gardner, M.; Church, R.; Ustin, S.; Scheer, G.; Green, R.O. Mapping chaparral in the Santa Monica Mountains using multiple endmember spectral mixture models. Remote Sens. Environ. 1998, 65, 267-279.

34. Schwieder, M.; Leitão, P.J.; Suess, S.; Senf, C.; Hostert, P. Estimating fractional shrub cover using simulated EnMAP data: A comparison of three machine learning regression techniques. Remote Sens. 2014, 6, 3427-3445. 
35. Camps-Valls, G. Machine learning in remote sensing data processing. In Proceedings of the 2009 IEEE International Workshop on Machine Learning for Signal Processing, Grenoble, France, 2-4 September 2009; pp. 1-6.

36. Vapnik, V.N. The Nature of Statistical Learning Theory; Springer-Verlag: New York, USA, 1995; Volume 8.

37. Pal, M.; Mather, P.M. Support vector machines for classification in remote sensing. Int. J. Remote Sens. 2005, 26, 1007-1011.

38. Huang, C.; Davis, L.S.; Townshend, J.R.G. An assessment of support vector machines for land cover classification. Int. J. Remote Sens. 2002, 23, 725-749.

39. Plaza, A.; Benediktsson, J.A.; Boardman, J.W.; Brazile, J.; Bruzzone, L.; Camps-Valls, G.; Chanussot, J.; Fauvel, M.; Gamba, P.; Gualtieri, A.; et al. Recent advances in techniques for hyperspectral image processing. Remote Sens. Environ. 2009, 113, 110-122.

40. Waske, B.; van der Linden, S.; Benediktsson, J.A.; Rabe, A.; Hostert, P. Sensitivity of support vector machines to random feature selection in classification of hyperspectral data. IEEE Trans. Geosci. Remote Sens. 2010, 48, 2880-2889.

41. Platt, J.C. Probabilistic outputs for support vector machines and comparison to regularized likelihood methods. Kernel Methods Support Vector Learn. 2000, 10, 61-74.

42. Wu, T.F.; Lin, C.J.; Weng, R.C. Probability estimates for multi-class classification by pairwise coupling. J. Mach. Learn. Res. 2004, 5, 975-1005.

43. Waske, B.; van der Linden, S. Classifying multilevel imagery from SAR and optical sensors by decision fusion. IEEE Trans. Geosci. Remote Sens. 2008, 46, 1457-1466.

44. Brown, M.; Lewis, H.G.; Gunn, S.R. Linear spectral mixture models and support vector machines for remote sensing. IEEE Trans. Geosci. Remote Sens. 2000, 38, 2346-2360.

45. Li, H.; Wang, Y.P.; Li, Y.; Wang, X.F. Pixel-unmixing moderate-resolution remote sensing imagery using pairwise coupling support vector machines: A case study. IEEE Trans. Geosci. Remote Sens. 2011, 49, 4298-4307.

46. Suess, S.; van der Linden, S.; Leitão, P.J.; Okujeni, A.; Waske, B.; Hostert, P. Import vector machines for quantitative analysis of hyperspectral data. IEEE Geosci. Remote Sens. Lett. 2014, 11, 449-453.

47. Leitão, P.J.; Moreira, F.; Osborne, P.E. Breeding habitat selection by steppe birds in Castro Verde: A remote sensing and advanced statistics approach. Ardeola 2010, 57, 93-116.

48. Moreira, F. Relationships between vegetation structure and breeding bird densities in fallow cereal steppes in Castro Verde, Portugal. Bird Study 1999, 46, 309-318.

49. Leitão, P.J.P. Improving Species Distribution Models to Describe Steppe Bird Occurence Patterns and Habitat Selection in Southern Portugal. Ph.D. Thesis, University of Southampton, Southampton, UK, December 2008.

50. Richter, R.; Schläpfer, D. Geo-atmospheric processing of airborne imaging spectrometry data. Part 2: Atmospheric/topographic correction. Int. J. Remote Sens. 2002, 23, 2631-2649.

51. Schläpfer, D.; Richter, R. Geo-atmospheric processing of airborne imaging spectrometry data. Part 1: Parametric orthorectification. Int. J. Remote Sens. 2002, 23, 2609-2630. 
52. Segl, K.; Guanter, L.; Rogass, C.; Kuester, T.; Roessner, S.; Kaufmann, H.; Sang, B.; Mogulsky, V.; Hofer, S. EeteS: an end-to-end image simulation tool applied to the EnMAP hyperspectral mission. IEEE J. Sel. Top. Appl. Earth Obs. Remote Sens. 2012, 5, 522-530.

53. Camps-Valls, G.; Bruzzone, L. Kernel-based methods for hyperspectral image classification. IEEE Trans. Geosci. Remote Sens. 2005, 43, 1351-1362.

54. Mountrakis, G.; Im, J.; Ogole, C. Support vector machines in remote sensing: A review. ISPRS J. Photogramm. Remote Sens. 2011, 66, 247-259.

55. Rabe, A.; van der Linden, S.; Hostert, P. ImageSVM, Version 3.0. Available online: Www.imagesvm.net 2014 (accessed on 12 August 2015).

56. Chang, C.C.; Lin, C.J. LIBSVM: A library for support vector machines. Acm Trans. Intell. Syst. Technol. 2011, 2, 27.

57. Van der Linden, S.; Rabe, A.; Held, M.; Jakimow, B.; Leitão, P.J.; Okujeni, A.; Schwieder, M.; Senf, C.; Suess, S.; Hostert, P. The EnMAP-Box-A toolbox and application programming interface for EnMAP data processing. Remote Sens. 2015, under review.

58. Powers, D.M. Evaluation: from precision, recall and F-measure to ROC, informedness, markedness and correlation. J. Mach. Learn. Technol. 2011, 2, 37-63.

59. Okujeni, A.; van der Linden, S.; Tits, L.; Somers, B.; Hostert, P. Support vector regression and synthetically mixed training data for quantifying urban land cover. Remote Sens. Environ. 2013, 137, 184-197.

60. Bioucas-Dias, J.M.; Plaza, A.; Dobigeon, N.; Parente, M.; Du, Q.; Gader, P.; Chanussot, J. Hyperspectral unmixing overview: Geometrical, statistical, and sparse regression-based approaches. IEEE J. Sel. Top. Appl. Earth Obs. Remote Sens. 2012, 5, 354-379.

61. Tukey, J.W. Exploratory Data Analysis; Wrigley, N., Bennet, R.J., Eds. Quantitative applications in the social sciences; Addison-Wesley: New Jersey, USA, 1977; Volume 2.

62. Melgani, F.; Bruzzone, L. Classification of hyperspectral remote sensing images with support vector machines. IEEE Trans. Geosci. Remote Sens. 2004, 42, 1778-1790.

63. Foody, G.M.; Mathur, A. The use of small training sets containing mixed pixels for accurate hard image classification: training on mixed spectral responses for classification by a SVM. Remote Sens. Environ. 2006, 103, 179-189.

64. Escadafal, R.; Huete, A. Improvement in remote sensing of low vegetation cover in arid regions by correcting vegetation indexes for soil noise. Comptes Rendus l'Académie des Sci.-Ser. II 1991, 312, 1385-1391.

65. Okin, G.S.; Roberts, D.A.; Murray, B.; Okin, W.J. Practical limits on hyperspectral vegetation discrimination in arid and semiarid environments. Remote Sens. Environ. 2001, 77, 212-225.

66. Mansour, K. Remote sensing based indicators of vegetation species for assessing rangeland degradation: Opportunities and challenges. Afr. J. Agric. Res. 2012, 7, 3261-3270.

67. Hellesen, T.; Matikainen, L. An object-based approach for mapping shrub and tree cover on grassland habitats by use of LiDAR and CIR orthoimages. Remote Sens. 2013, 5, 558-583.

(C) 2015 by the authors; licensee MDPI, Basel, Switzerland. This article is an open access article distributed under the terms and conditions of the Creative Commons Attribution license (http://creativecommons.org/licenses/by/4.0/). 